\title{
Charge Transport Studies on Novel PT-derivatives with Hydrophilic Anchoring Groups
}

\author{
Mathias Nyman $^{1}$, Kenta Akitsu ${ }^{2}$, Naoki Otani ${ }^{3}$, Mitsunobu Matsumura ${ }^{3}$, Takaya \\ $\mathrm{Kubo}^{2}$, Hiroshi Segawa ${ }^{2}$, and Ronald Österbacka ${ }^{1}$ \\ ${ }^{1}$ Physics/Department of Natural Sciences and Center for Functional Materials, Åbo Akademi \\ University, Porthansgatan 3, 20500 Turku, Finland \\ ${ }^{2}$ Research Center for Advanced Science and Technology, The University of Tokyo, 4-6-1, \\ Komaba, Meguro-ku, Tokyo 153-8904, Japan \\ ${ }^{3}$ Synthesis Research Department, Nissan Chemical Industry, Ltd., 722-1, Tsuboi-cho, \\ Funabashi-shi, Chiba 274-8807, Japan
}

Corresponding author: M. Nyman

Tel.: +35822154607

Fax: +35822154776

E-mail: mathnyma@abo.fi

Keywords: polymer, hybrid solar cell, charge transport, photo-CELIV

\begin{abstract}
Using charge extraction techniques we have clarified the charge transport properties of novel poly-thiophene derivatives intended for use as sensitizers in polymer sensitized solar cells. The polymers are of low molecular weight in order to enable penetration into a mesoporous titanium dioxide film. Hydrophilic anchoring units are attached to the thiophene ring to facilitate attachment of the polymer to the titanium dioxide. The hole transporting properties were determined in order to clarify the possibility of using the polymers both as sensitizers and hole transporting material in a hybrid type device. We found that the mobilities are in the range of $1-4 \cdot 10^{-8} \mathrm{~cm}^{2} / \mathrm{Vs}$. The low mobility is compensated by very long charge carrier lifetimes in the order of $100 \mathrm{~ms}$ resulting in a very promising charge carrier drift distance of 1 $\mu \mathrm{m}$.
\end{abstract}




\section{Introduction}

Dye sensitized solar cells (DSSC) holds great promise for future cost-efficient and environmentally friendly energy production. DSSC technology has improved rapidly since the first DSSC was presented in 1991 [1] with efficiencies increasing from 7,9\% to $\sim 11,0 \%$ [2]. However, further improvement of the efficiency and the lifetime of the cells remains a challenge. In addition, the highest efficiency DSSCs employ liquid electrolytes as hole transporting materials which are not as stable as solid state cells due to possible leakage and evaporation of solvent. The s.c. $\pi$-conjugated polymers have been used as possible solid-state hole transporting materials $[3,4]$. However, the efficiencies are still low compared to the state-of-the-art liquid electrolyte DSSCs. In order to improve the efficiencies of these types of devices some key properties need to be improved.

The most important properties when using polymers as a solid state hole transporting material is good pore-filling into the mesoporous $\mathrm{TiO}_{2}$ layer and that the polymer has a sufficiently high mobility in order to avoid losses due to charge carrier recombination [5, 6]. Polymers are typically large and bulky molecules so in order to achieve good pore-filling it is essential to have a low molecular weight. However, it has been shown that the mobility goes down with the molecular weight [7] suggesting that it may be challenging to incorporate both a good pore-filling and high mobility in the same material. The use of polymers as sensitizing materials has also been demonstrated as well as using the same polymer both as sensitizing and hole transporting material $[8,9]$.

When using polymers as sensitizers it is highly important that the polymers attach efficiently enough to the mesoporous $\mathrm{TiO}_{2}$ layer. Recently Kubo et al used hydrolysis to create hydrophilic carboxyl acid groups attached directly to the thiophene ring to ensure good adhesion [10]. It was shown that the hydrophilic anchoring units improved the solar cell performance. The polymers were of low molecular weight to enable both good pore-filling and $\mathrm{TiO}_{2}$ coverage. In order to determine the viability of using the PT-derivatives also as a hole transporting layer the charge transport properties need to be clarified. Photo-CELIV (Charge Extraction by a Linearly Increasing Voltage) is a valuable tool when studying the charge transport properties of disordered organic materials since it is possible to measure both the mobility and photo generated charge concentrations simultaneously [11]. In this work photo-CELIV is used to measure the mobility of the PT-derivatives. By varying the hydrolysis ratio we seek to clarify if the hydrolysis has any effect on the transport properties. 


\section{Material and Methods}

A random copolymer poly[(methylthiophene-3yl-carboxylate)-ran-(thiophene)] (PT-MC) was synthesized by dehalogenation polymerization of a 2:1 mixture of diethyl 2,5dibromothiophene-3-ylcarboxylate and 2,5-dibromothiophene in the presence of $\mathrm{Ni}(\mathrm{cod})_{2}$ catalyst. PT-MCs with a mean molecular weight $\left(\mathrm{M}_{\mathrm{W}}\right)$ of 2300 and polydispersity index (PDI) of 1,56 were obtained using a silica gel column. Silica gel chromatography with chloroform as eluent is used to obtain the $\mathrm{M}_{\mathrm{W}}$. Polythiophene derivatives with carboxylic acid units (dubbed as PT-C) were synthesized by hydrolysis of PT-MCs. Hydrolysis ratios (HRs) were determined in a deuterated DMSO by 1H-NMR. Detailed synthesis scheme of the polymers were given elsewhere [10].

Samples were prepared as follows; ITO covered borosilicate glass (from Präzisions Glas \& Optik $\mathrm{GmbH}$ ) was used as substrate. Half of the substrate was etched with aqua regia for roughly 40 minutes. The substrates were subsequently cleaned in a 1:1:5 blend of $\mathrm{H}_{2} \mathrm{O}_{2}, \mathrm{NH}_{3}$ and water in an ultra sonicator at $80^{\circ} \mathrm{C}$ for 20 minutes. The substrates were then transferred into a nitrogen atmosphere glovebox where a $20 \mathrm{~nm}$ layer of Au was thermally evaporated on the ITO (to prevent the hydrophilic PT-derivatives to attach to the ITO). The PT-derivatives were then drop-casted from a $10 \mathrm{mg} / \mathrm{ml}$ chloroform solution. The polymer films were allowed to dry overnight. Finally a $5 \mathrm{~nm}$ blocking layer of LiF was evaporated on the polymer film followed by a $65 \mathrm{~nm}$ layer of Al. The samples were transferred to a cryostat outside the glovebox, the samples were kept at a vacuum of $\sim 10^{-6}$ mbar or better for several hours to draw out any eventual oxygen contamination.

Photo-CELIV measurements were carried out as reported earlier [12]. A sample is kept at flat band conditions by an offset voltage, $V_{O F F}$, and is excited by a $6 \mathrm{~ns}$ laser pulse at $532 \mathrm{~nm}$ through the semitransparent Au electrode. After a variable delay time a linearly increasing voltage pulse is applied to extract the photo generated charges ( $A=$ max voltage/pulse length). The laser intensity per pulse was $2,5 \mathrm{~mJ} / \mathrm{cm}^{2}$ unless otherwise stated. From the displacement current $(j(0)$, the initial step in the current transient) the thickness of the device can be calculated [13]. The thicknesses of the samples were between 230 and $530 \mathrm{~nm}$, the large differences being due to the drop casting process. From the time it takes for the charge current transient to reach its maximum value, $t_{\max }, j(0)$ and $\Delta j$ (the maximum current minus $j(0)$ ) one can calculate the mobility. The mobility has been calculated taking the generation profile into account according to the recipe given by Juška et al [14]. 
In order to make sure there would not be a build-up of charges the delay time between consecutive measurements (the transients were averaged $\sim 100$ times) was at least five seconds. No drift in the transients was seen during the course of the averaging and the samples showed stabile behavior over several days. 


\section{Results and discussion}

The synthesis scheme and energy levels of the PT-derivatives are given in Figure 1. The PTMC polymer is fully methylated (no carboxylic acid groups). The PT-C-39 and PT-C-53 has $39 \%$ and $53 \%$ carboxylic acid vs. methyl units respectively. The absorption spectra of the studied polymers are shown in Figure 2. The spectra show featureless absorption indicating inhomogeneously broadened bands typically observed in disordered organic semiconductors. The spectra are fairly similar, the notable difference being that the spectrum for PT-C-53 is slightly narrower than the other two.

Figure 3 shows a typical photo-CELIV current transient response saturated on light intensity, all polymers show similar behavior. A small dark conductivity can be seen, however, this should not have a large effect on the results since the dark current is much smaller than the light induced current transient. The $\Delta j$ is at most as large as $j(0)$ which implies Langevin recombination [14]. The $t_{\max }$ is clearly observable allowing for the mobility to be calculated between $1-4 \cdot 10^{-8}$ at room temperature for all the polymers in this study. The rather low mobility was to be expected due to the low molecular weight [7]. No significant time dependence was seen on the mobility in the time scales studied.

The electric field dependence on the mobility for different temperatures is shown in Figure 4. The temperature range is limited due to the fact that the transport becomes very slow at low temperatures making the measurements difficult to perform. The mobility does not show any clear dependence on the electric field at the temperatures and fields studied in any of the polymers. However, due to the limited field region and large uncertainties caused by the low mobility it should be stressed and that a weak dependence on the electric field cannot be excluded.

The mobility dependence on $1 / T$ is shown in Figure 5. The mobilities are taken as an average of the mobilities at different electric fields and not at zero electric field, this is a fair approximation as the field dependence is weak. From the Arrhenius plot the activation energy $\Delta E$ is estimated to $44 \mathrm{meV}$ for PT-MC, $25 \mathrm{meV}$ for PT-C-39 and $58 \mathrm{meV}$ for PT-C-53. Surprisingly, PT-C-39 show a significantly weaker temperature dependence than the other polymers. In addition, PT-C-39 has the lowest mobility at room temperature. Craciun et al showed by studying a number of different materials that the bulk-limited charge transport in disordered organic semiconductors can be generalized into an Arrhenius type temperature dependence $\mu_{h}=\mu_{0} \exp (-\Delta E / k T)$ with $\mu_{0}$ being a universal mobility of $30-40 \mathrm{~cm}^{2} / \mathrm{Vs}$ and $\Delta E$ the activation energy meaning that low mobility implies large activation energy and vice versa 
[16]. Clearly the materials studied here do not fit into this picture since both the mobility and activation energies are low.

This can be understood in the picture of Miller-Abrahams hopping rates. Charge transport in disordered organic materials is typically modeled by hopping transport in a Gaussian density of states. For describing the hopping rate $W_{i j}$ between sites $i$ and $j$ the Miller-Abrahams formalism is frequently used [12]:

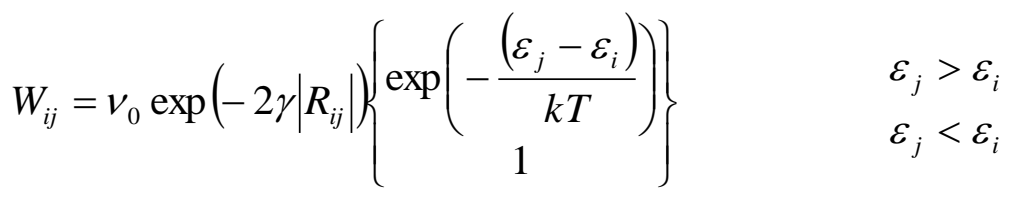

where $v_{0}$ is the phonon vibration frequency, $\gamma$ is the inverse localization radius (the inverse of the wavefunction overlap integral), $R_{i j}$ is the intersite distance, $\varepsilon_{j}$ is the energy of site $j, k$ is the Boltzman constant and $T$ is the temperature. The hopping rate consists of a temperature independent part, $v_{0} \exp \left(-2 \gamma\left|R_{i j}\right|\right)$ and a temperature dependent part $\exp \left(-\left(\varepsilon_{j}-\varepsilon_{i}\right) / k T\right)$. The weak temperature dependence implies small energetic disorder. This means that the reason for the low mobility most probably is long hopping distances since the transport is governed by the rate of the longest jump. The long hopping distances are probably due to low packing of the low-molecular weight polymer chains and/or small aggregate. PT-C-39 has the lowest room temperature mobility and the weakest temperature dependence which implies longest hopping distances and lowest packing density resulting in the temperature independent part dominating over the temperature dependent part. This can be understood since the polar carboxylic acid groups will not easily form tight packed structures with the thiophene back-bone. In addition, by increasing the hydrolysis ratio the activation energy increases, possibly due to the formation of a different packing density when more carboxylic acid groups are present in the material. In order to improve the mobility one should seek ways of improving the packing density, i.e. increasing the wave-function overlap factor without losing the good pore filling and adhesion properties.

Figure 6 shows the calculated charge densities as a function of delay time for all three polymers. One should note that the current transients do not quite saturate to the dark response, which will lead to a slight underestimation of the generated and extracted charge density. The half-life of the charge carriers is roughly $100 \mathrm{~ms}$ for all polymers, indicating very slow recombination. A good measure of a hole transporting layer is the product of the mobility $\mu$ with the lifetime $\tau$, taking $\mu \sim 2 \cdot 10^{-8} \mathrm{~cm}^{2} / \mathrm{Vs}$ and $\tau \sim 100 \mathrm{~ms}$ gives a $\mu \tau$-product of 
$2 \cdot 10^{-9} \mathrm{~cm}^{2} / \mathrm{V}$. The average drift distance $l$ can be estimated by multiplying the $\mu \tau$-product with a typical electric field $(l=\mu \tau F)$. Taking $F=5 \cdot 10^{4} \mathrm{~V} / \mathrm{cm}$ (corresponding to $1 \mathrm{~V}$ over $200 \mathrm{~nm}$ ) gives an average drift distance of $1 \mu \mathrm{m}$. This shows that the observed low mobility is compensated by long charge carrier lifetimes (slow recombination) to allow for a reasonably high average drift distance typically only seen in bulkheterojunction blends.

\section{Conclusions}

The charge transport properties of three novel PT-derivatives have been studied. The mobilities are generally very low, in the order of $10^{-8} \mathrm{~cm}^{2} / \mathrm{Vs}$, however, the long charge carrier lifetimes and the average drift distance of $1 \mu \mathrm{m}$ are encouraging. The low mobility is most likely due to long hopping distances due to low packing density. In order to improve the mobility one should seek ways of improving the packing density without losing the good pore filling and adhesion properties.

\section{Acknowledgements}

Academy of Finland projects \#135262 and \#137093 are acknowledged for funding. This research is supported by the Japan Society for the Promotion of Science (JSPS) through its "Funding Program for World-Leading Innovative R\&D on Science and Technology (FIRST Program)". Simon Sandén and prof. Gytis Juška are acknowledged for valuable discussions. 


\section{References}

[1] B. O'Regan, and M. Grätzel, A low-cost, high-efficiency solar cell based on dyesensitized colloidal $\mathrm{TiO}_{2}$ films, Nature 353 (1991) 737-740

[2] M. A. Green, K. Emery, Y. Hishikawa, W. Warta, E. D. Dunlop, Solar cell efficiency tables (version 40), Prog. Photovolt: Res. Appl. 20 (2012) 606-614

[3] K. Murakoshi, R. Kogure, Y. Wada, and S. Yanagida, Solid State Dye-Sensitized $\mathrm{TiO}_{2}$ Solar Cell with Polypyrrole as Hole Transport Layer, Chem. Lett. 5 (1997) 471-472

[4] D. Gebeyehu, C. J. Brabec, N. S. Sariciftci, D. Vangeneugden, R. Kiebooms, D. Vanderzande, F. Kienberger, H. Schindler, Hybrid solar cells based on dye-sensitized nanoporous $\mathrm{TiO}_{2}$ electrodes and conjugated polymers as hole transport materials, Synth. Met. 125 (2002) 279-287

[5] A. F. Nogueira, C. Longo, M.-A. De Paoli, Polymers in dye sensitized solar cells: overview and perspectives, Coord. Chem. Rev. 248 (2004) 1455-1468

[6] A. Hagfeldt, G. Boschloo, L. Sun, L. Kloo, H. Pettersson, Dye-Sensitized Solar Cells, Chem. Rev. 110 (2010) 6595-6663

[7] C. Goh, R. J. Kline, M. D. McGehee, E. N. Kadnikova, J. M. J. Fréchet, Molecularweight-dependent mobilities in regioregular poly(3-hexyl-thiophene) diodes, Appl. Phys. Lett. 86 (2005) 122110

[8] T. J. Savenije, J. M. Warman, A. Goossens, Visible light sensitisation of titanium dioxide using a phenylene vinylene polymer, Chem. Phys. Lett. 287 (1998) 1-2 148153

[9] S. Spiekermann, G. Smestad, J. Kowalik, L. M. Tolbert, and M. Grätzel, Poly(4undecyl-2,2'-bithiophene) as a hole conductor in solid state dye sensitized titanium dioxide solar cells, Synth. Met. 121 (2001) 1603-1604

[10] T. Kubo, K. Akitsu, S. Uchida, H. Segawa, N. Otani, M. Tomura, T. Tamura, M. Matsumura, Polymer-sensitized Solar Cells with Novel Soluble Polythiophene Derivatives, J. Photopolym. Sci. Technol. 332 (2010) 283-286

[11] G. Juška, M. Viliūnas, K. Arlauskas, N. Nekrašas, N. Wyrsch, and L. Feitknecht, Hole drift mobility in $\mu \mathrm{c}-\mathrm{Si}: \mathrm{H}$, J. Appl. Phys. 89 (2001) 4971-4974

[12] A. Pivrikas, N. S. Sariciftci, G. Juška, and R. Österbacka, A Review of Charge Transport and Recombination in Polymer/Fullerene Solar Cells, Prog. Photovolt: Res. Appl. 15 (2007) 677-696

[13] G. Juška, K. Arlauskas, M. Viliūnas, and J. Kocka, Extraction Current Transients: New Method of Study of Charge Transport in Microcrystalline Silicon, Phys. Rev. Lett. 84 (2000) 4946-4949 
[14] G. Juška, N. Nekrašas, V. Valentinavičius, P. Meredith, and A. Pivrikas, Extraction of photogenerated charge carriers by linearly increasing voltage in the case of Langevin recombination, Phys. Rev. B 84 (2011) 155202

[15] M. Pope and C. E. Swenberg, Electronic Processes in Organic Crystals and Polymers, $2^{\text {nd }}$ ed. (Oxford University Press, New York, 1999)

[16] N. I. Craciun, J. Wildeman, and P. W. M. Blom, Universal Arrhenius Temperature Activated Charge Transport in Diodes from Disordered Organic Semiconductors, Phys. Rev. Lett. 100 (2008) 056601 


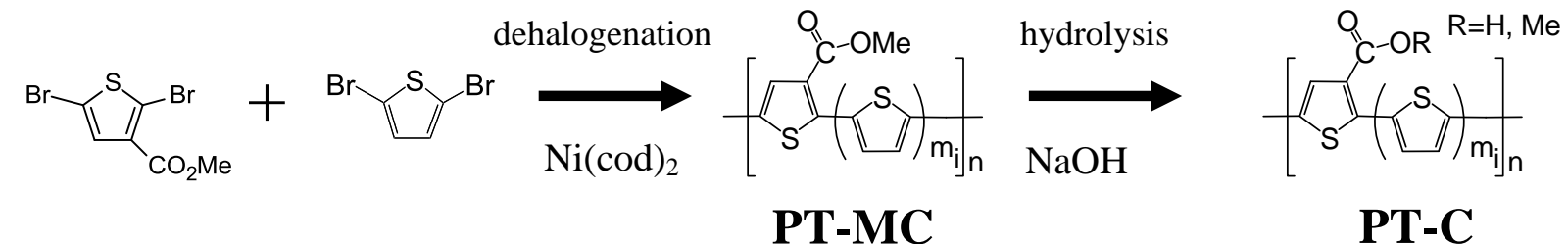

$$
\mathrm{m}_{\mathrm{i}} \geq 0, \frac{\sum_{\mathrm{i}=1}^{\mathrm{n}} \mathrm{m}_{\mathrm{i}}}{\mathrm{n}} \fallingdotseq 0.5
$$

\begin{tabular}{cccc} 
Sample & $\begin{array}{c}\text { Hydrolysis } \\
\text { ratio, \% }\end{array}$ & $\begin{array}{c}\text { HOMO, } \\
\text { eV }\end{array}$ & $\begin{array}{c}\text { LUMO, } \\
\text { eV }\end{array}$ \\
\hline PT-C-39 & 39 & -5.2 & -3.1 \\
PT-C-41 & 41 & -5.2 & -3.1 \\
PT-C-53 & 53 & -5.4 & -3.3 \\
\hline
\end{tabular}

Figure 1 Synthesis scheme and energy levels of the PT-derivatives

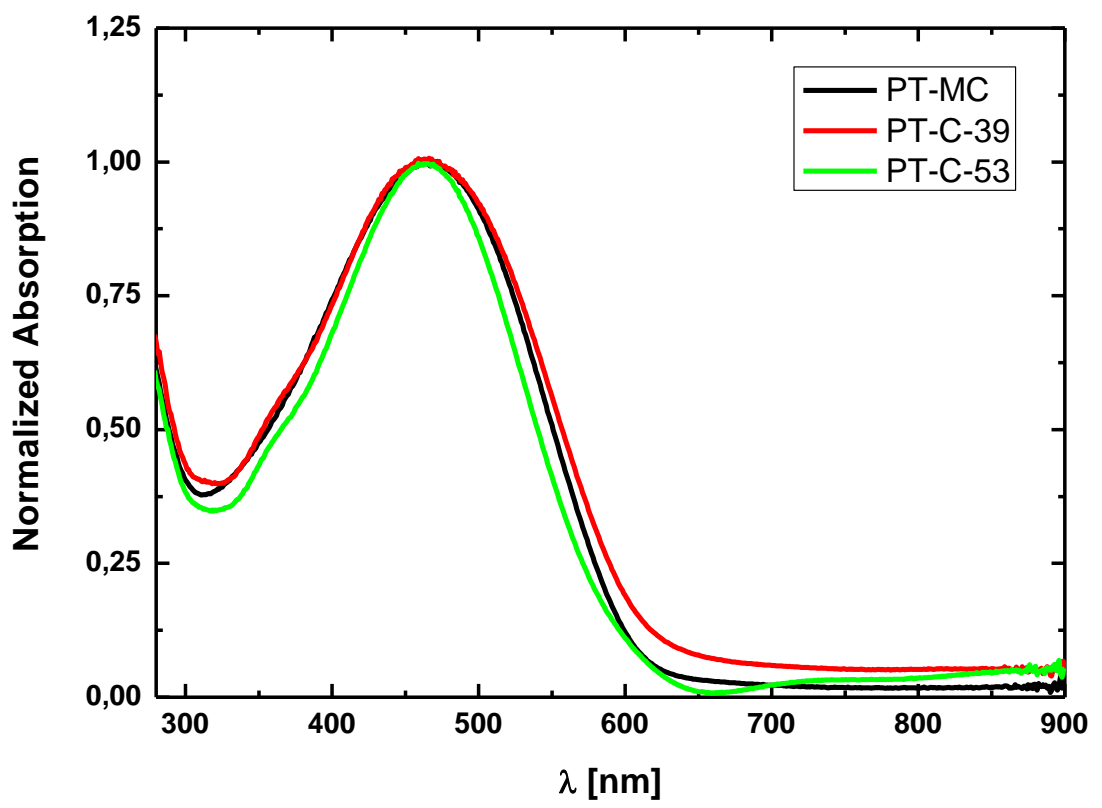

Figure 2 Normalized absorption spectra of three PT-derivatives 


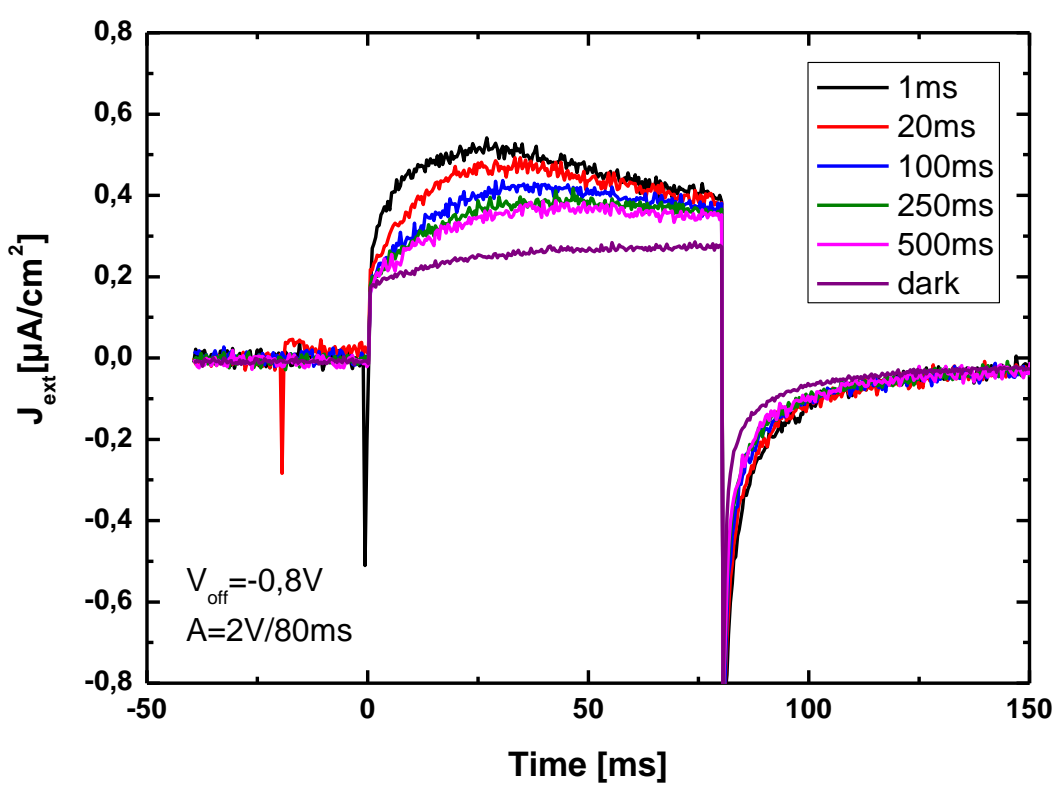

Figure 3 Photo-CELIV currents transients for different delay times of a PT-MC device. The laser intensity per pulse was $8 \mathrm{~mJ} / \mathrm{cm}^{2}$.

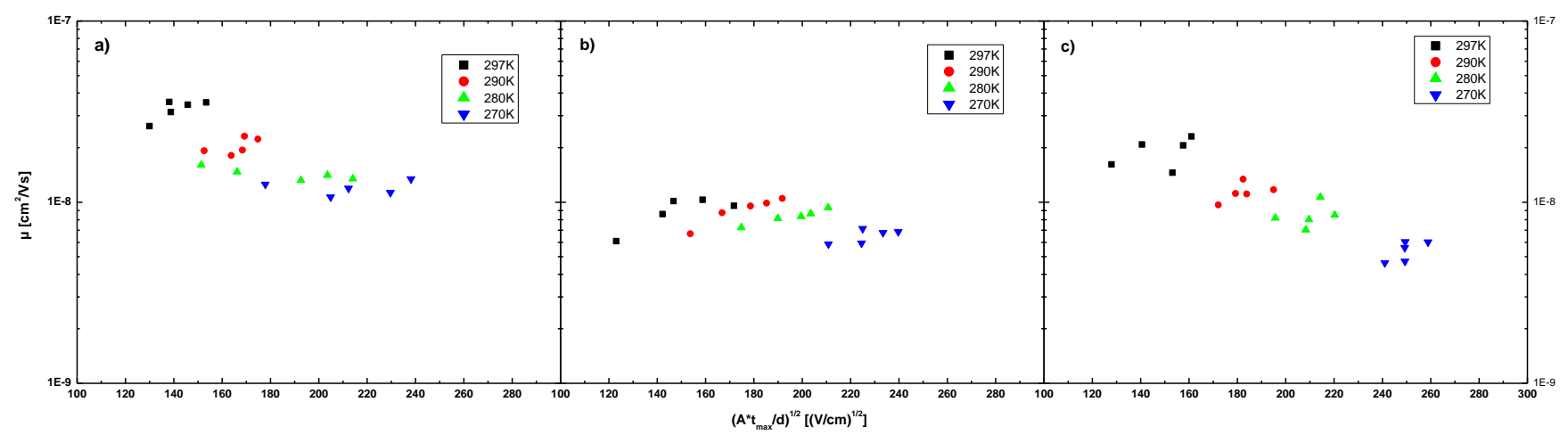

Figure 4 The mobility as a function of electric field at different temperatures for a) a PT-MC device b) a PT-C-39 device and c) a PT-C-53 device. 


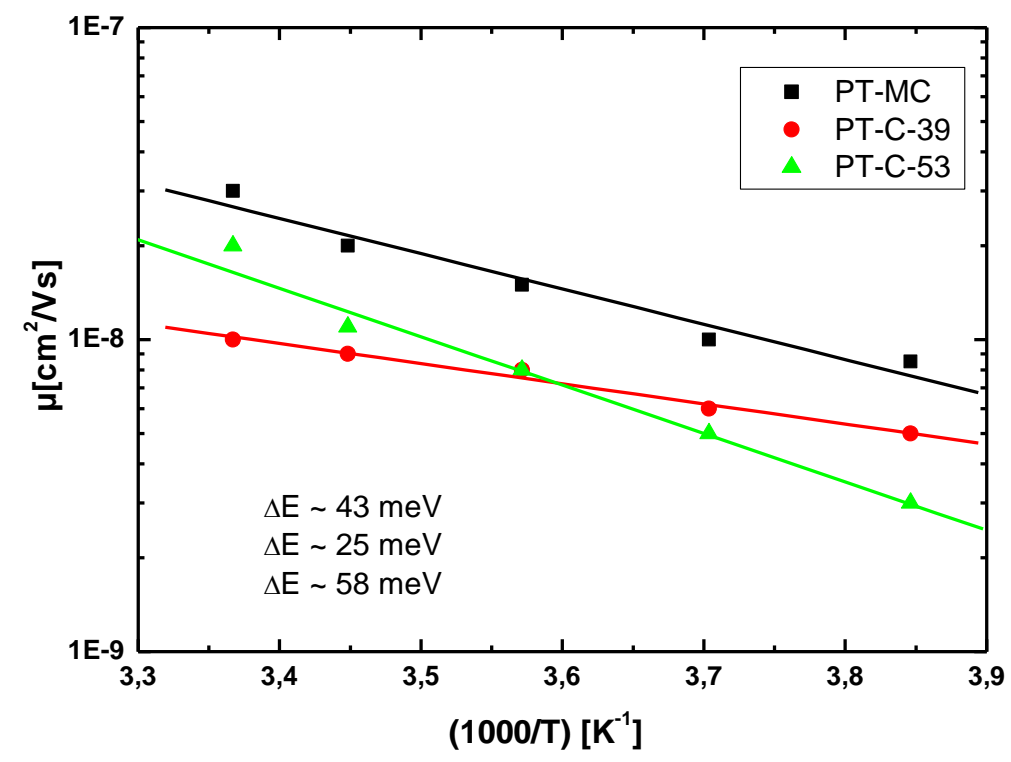

Figure 5 Arrhenius plot for three different PT-derivatives

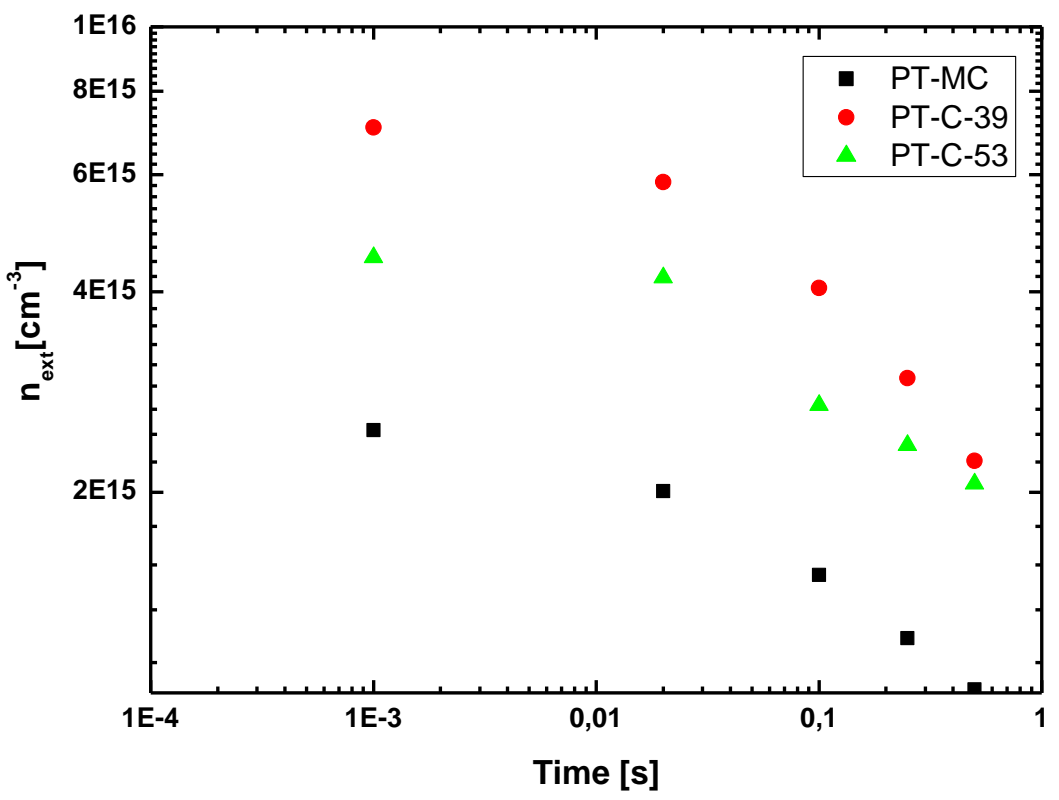

Figure 6 The concentrations of extracted charges in three different PT-derivatives. The laser intensity per pulse was $8 \mathrm{~mJ} / \mathrm{cm}^{2}$. 

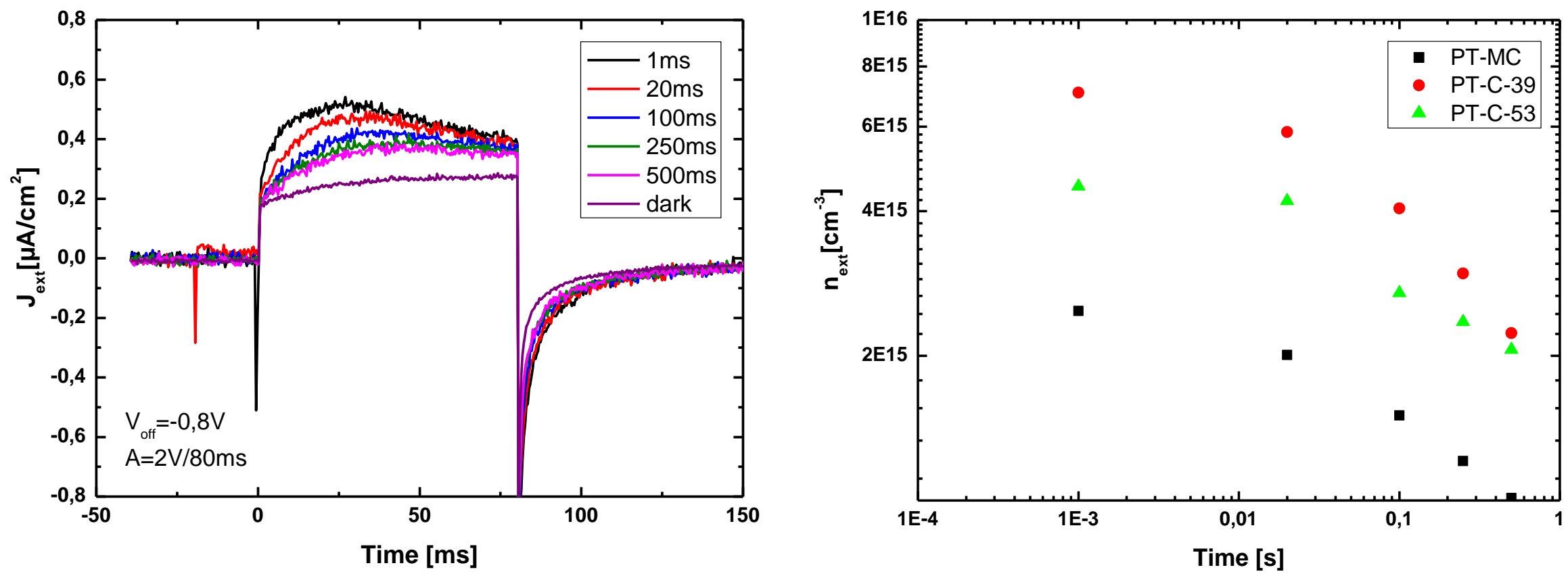